\title{
Multi-column parallel QC- LDPC decoder architecture for NAND flash memory
}

\author{
Wei Shen ${ }^{1}$, Cheng Chen ${ }^{2}$, and Jin Sha ${ }^{3 a)}$ \\ ${ }^{1}$ School of Electrical Science and Engineering, Nanjing University, \\ Nanjing 210046, People's Republic of China \\ ${ }^{2}$ Microelectronics R\&D Institute, ZTE Corporation, \\ Nanjing 210046, People's Republic of China \\ ${ }^{3}$ Shenzhen Research Institute, Nanjing University, \\ Nanjing 210046, People's Republic of China \\ a)shajin@nju.edu.cn
}

Abstract: Quasi-cyclic (QC) low-density parity-check (LDPC) codes are famous for their excellent error correction performance and hardware friendly structure in NAND flash memory application. Array LDPC code is a type of highly structured QC-LDPC code that provides a good balance between performance and complexity. In this paper, a method is proposed for the construction of $(18900,17010)$ LDPC code that is based on the Latin square and an improved array dispersion strategy to achieve multi-column alignment of the structure. Compared with traditional design, the parallel hardware architecture reduces the number of barrel shifters by $32 \%$. The corresponding ASIC implementation results show that the throughput of the proposed QC-LDPC code was up to $3.49 \mathrm{~Gb} / \mathrm{s}$ and the throughput-to-area (TAR) of the proposed codes was significantly improved.

Keywords: QC-LDPC, NAND flash memory, column-based shuffle decoding, multiple-columns

Classification: Integrated circuits

\section{References}

[1] G. Dong, et al:: "Using data postcompensation and predistortion to tolerate cell-to-cell interference in MLC NAND flash memory," IEEE Trans. Circuits Syst. I, Reg. Papers 57 (2010) 2718 (DOI: 10.1109/TCSI.2010.2046966).

[2] K. Kim: "Technology for sub-50 nm DRAM and NAND flash manufacturing," IEEE International Electron Devices Meeting, 2005. IEDM Technical Digest (2005) 323 (DOI: 10.1109/IEDM.2005.1609340).

[3] J. Li, et al.: "Realizing unequal error correction for NAND flash memory at minimal read latency overhead," IIEEE Trans. Circuits Syst. II, Exp. Briefs 61 (2014) 354 (DOI: 10.1109/TCSII.2014.2312640).

[4] R. Gallager: "Low-density parity-check codes," IRE Trans. Inf. Theory 8 (1962) 21 (DOI: 10.1109/TIT.1962.1057683).

[5] M. P. C. Fossorier, et al.: "Reduced complexity iterative decoding of lowdensity parity check codes based on belief propagation," IEEE Trans. Commun. 47 (1999) 673 (DOI: 10.1109/26.768759). 
[6] K. C. Ho, et al.: "A 520k $(18900,17010)$ array dispersion LDPC decoder architectures for NAND flash memory," IEEE Trans. Very Large Scale Integr. (VLSI) Syst. 24 (2016) 1293 (DOI: 10.1109/TVLSI.2015.2464092).

[7] H. C. Lee, et al.: "Optimization techniques for the efficient implementation of high-rate layered QC-LDPC decoders," IEEE Trans. Circuits Syst. I, Reg. Papers 64 (2017) 457 (DOI: 10.1109/TCSI.2016.2612655).

[8] J. Li, et al.: "Algebraic quasi-cyclic LDPC codes: Construction, low error-floor, large girth and a reduced complexity decoding scheme," IEEE Trans. Commun. 62 (2014) 2626 (DOI: 10.1109/TCOMM.2014.2339329).

[9] M. R. Li, et al.: "A low-complexity LDPC decoder for NAND flash applications," 2014 IEEE International Symposium on Circuits and Systems (ISCAS) (2014) 213 (DOI: 10.1109/ISCAS.2014.6865103).

[10] E. Eleftheriou and S. Olcer: "Low-density parity-check codes for digital subscriber lines," IEEE Communication Letters (2002) 1752 (DOI: 10.1109/ ICC.2002.997149).

[11] W. Shao, et al:: "Dispersed array LDPC codes and decoder architecture for NAND flash memory,” IEEE Trans. Circuits Syst. II, Exp. Brief (DOI: 10. 1109/TCSII.2017.2783438).

\section{Introduction}

NAND flash memories are widely used for storage in mobile devices. Owing to the growing demand for high-density storage capacity and throughput, multi-level cell (MLC) and trinary-level cell (TLC) [1,2] techniques are used in flash memory. However, data reliability is reduced due to higher raw bit error rates (RBER) introduced by the increasing bit density [3].

Error correction codes (ECC), such as BCH codes and LDPC codes are efficient approaches to guarantee the data reliability. Compared with $\mathrm{BCH}$ codes, LDPC codes yield superior error correction performance with parity bits of the same size $[4,5]$. QC-LDPC code is known for its hardware-friendly structure and excellent error correction performance. Several studies contributed on the implementation of the QC-LDPC codes $[6,7,8,9]$, including improving the decoding algorithm and optimizing hardware architecture. Few studies, however, have focused on increasing the throughput of the decoding architecture. In this paper, we aim at developing a parallel decoding hardware architecture to meet the throughput requirements of both the Toggle DDR 2.0 and the ONFI 3.0 NAND interfaces [10].

The remainder of this paper is organized as follows. Section 2 introduces the column-based shuffle decoding algorithm, along with the challenges to parallel implementation. We also propose here the construction of a $(18900,17010)$ QCLDPC code. In Section 3, the overall parallel architecture and the results of its implementation are detailed. Finally, we offer our conclusions in Section 4.

\section{Code construction and decoding algorithm}

\subsection{Column-based shuffle decoding (CBSD) algorithm}

An $(N, N-M)$ LDPC code represents the parity-check matrix $H$ of size $M \times N$. At the $w$ th iteration, the check-to-variable $(\mathrm{C} 2 \mathrm{~V})$ message from check node $(\mathrm{CN}) c$ to variable node $(\mathrm{VN}) v$ and the variable-to-check (V2C) message from $\mathrm{VN} v$ to $\mathrm{CN} c$ 
are denoted by $L_{c v}^{w}$ and $L_{v c}^{w}$ respectively, where $c=0,1, \ldots, M-1$, and $v=$ $0,1, \ldots, N-1$. Note that $N(c)$ is the set of VNs connected to CN $c$ and $M(v)$ the set of CNs connected to VN $v$. The VNs are divided equally into $G$ groups and each group $N_{g}$ contains $N / G$ VNs, where $g=0,1, \ldots, G-1$. The following shows the message update between the CNs and VNs in the gth group in the wth iteration.

1. VNU operation: The V2C message $L_{v c}^{w}$ is updated as in Eq. (1).

$$
L_{v c}^{w}=L_{\text {init }, v}+\sum_{c^{\prime} \in M(v) \backslash c} L_{c^{\prime} v}^{w-1}
$$

$L_{\text {init }, v}$ is the initial channel value of $\mathrm{VN} v$.

2. CNU operation: The $\mathrm{C} 2 \mathrm{~V}$ message $L_{c v}^{w}$ is updated as in Eq. (2), and a represents the scaling factor. $N_{g}$ is the set of VNs located in the gth groups for $g=0,1, \ldots, G-1 . N_{L}$ is the union of $N_{0}, N_{1}, \ldots, N_{g-1}$, and $N_{R}$ is the union of $N_{g}, N_{g+1}, \ldots, N_{G-1}$.

$$
\begin{aligned}
L_{c v}^{w}= & a \times \prod_{v^{\prime} \in N(c) \cap N_{L}} \operatorname{sgn}\left(L_{v^{\prime} c}^{w}\right) \prod_{v^{\prime} \in N(c) \cap N_{R} \backslash v} \operatorname{sgn}\left(L_{v^{\prime} c}^{w-1}\right) \\
& \times \min \left(\min _{v^{\prime} \in N(c) \cap N_{L}}\left|L_{v^{\prime} c}^{w}\right|, \min _{v^{\prime} \in N(c) \cap N_{R} \backslash v}\left|L_{v^{\prime} c}^{w-1}\right|\right)
\end{aligned}
$$

The posteriori probability $L_{a p p, v}$ is computed as in Eq. (3) and used for hard decision.

$$
L_{a p p, v}=L_{i n i t, v}+\sum_{c^{\prime} \in M(v)} L_{c^{\prime} v}^{w-1}
$$

\subsection{Parallelism analysis}

Ho et al. proposed a top-down design that encapsulates optimization as well as hardware implementation. The architecture can achieve a high throughput with the degree-of-parallelism (DOP) at one. The matrix $H^{Q C}$ consists of four column groups as shown in Fig. 1. Note that each element of $\left\{\alpha^{A}, \alpha^{B}, \alpha^{C}, \alpha^{D}\right\}$ represents the submatrix and each element of $\{A, B, C, D\}$ represents the shifting value of the corresponding submatrix cycle shifted by an identity matrix of size $z$. The submatrix is an all-zero matrix when the element is zero. Barrel shifters are widely used to align the $\mathrm{C} 2 \mathrm{~V}$ messages of column groups. The $\mathrm{C} 2 \mathrm{~V}$ and $\mathrm{V} 2 \mathrm{C}$ messages in

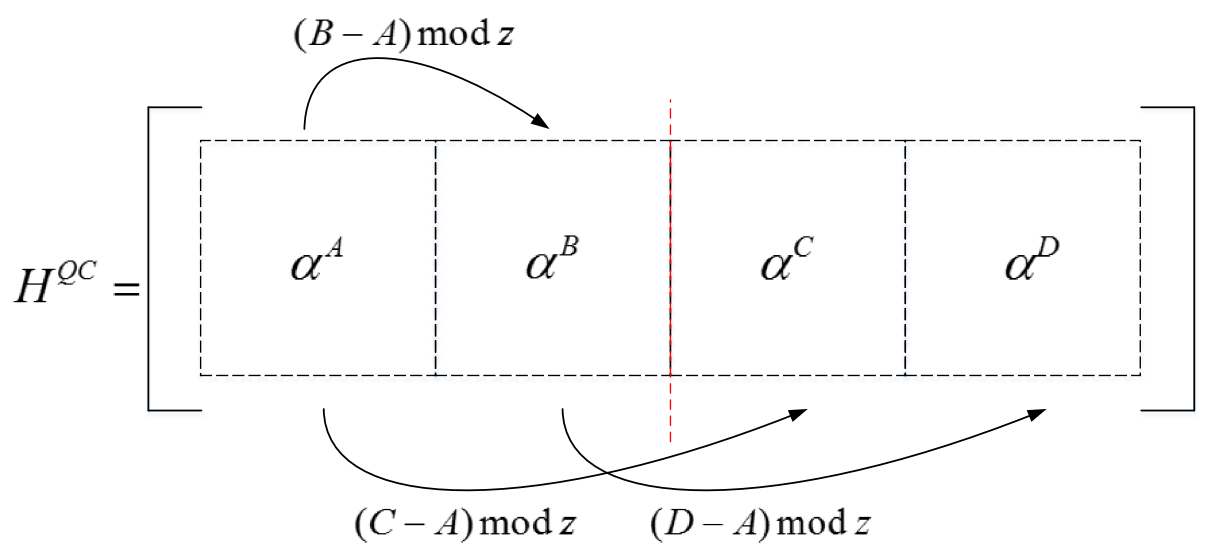

Fig. 1. Shifting parameter of barrel shifters 


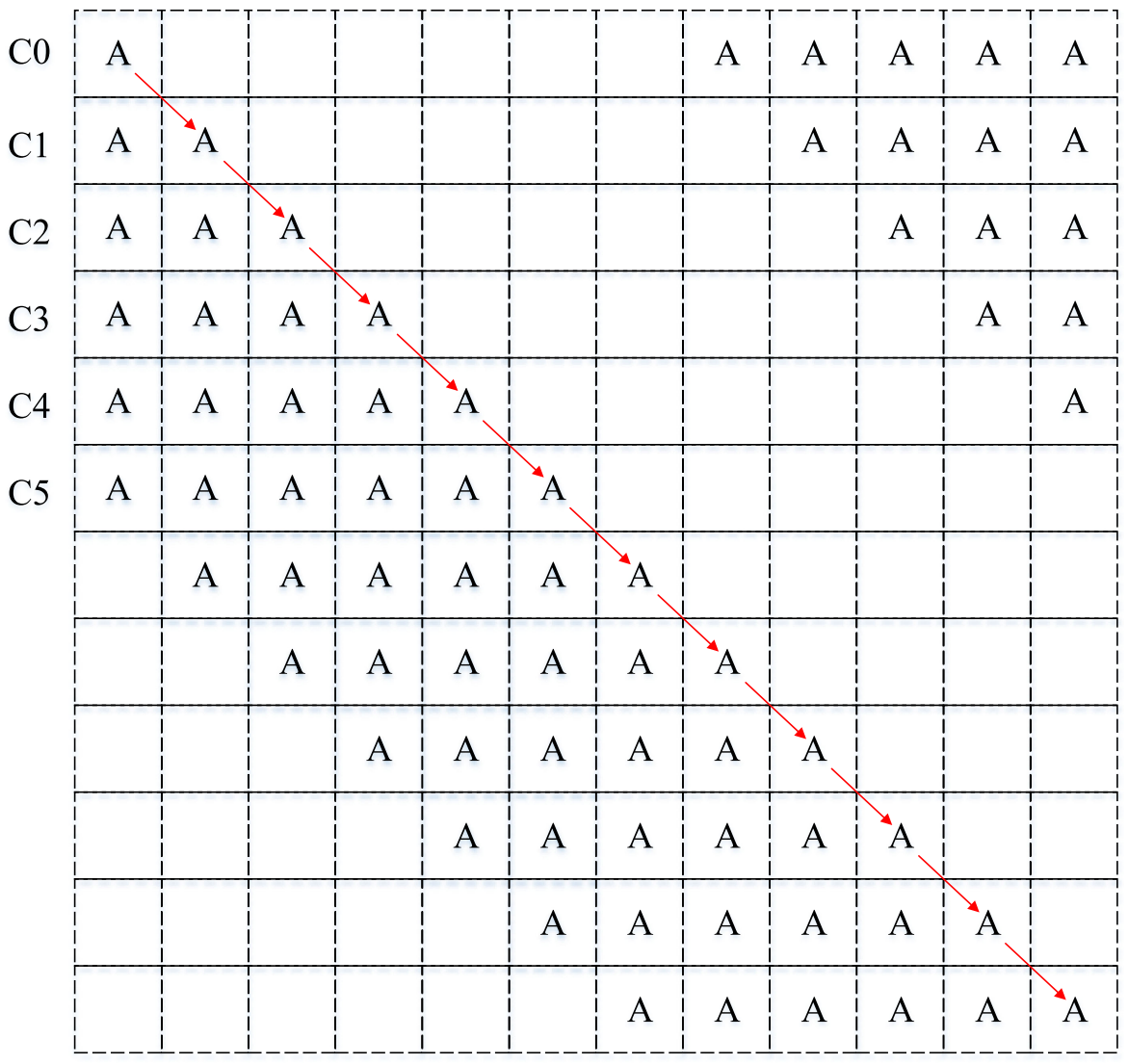

Fig. 2. Behavior of $\mathrm{CNU}$ block processing units

these four column groups are updated one by one. To align $\alpha^{A}$ with $\alpha^{B}$, the shifting parameter of the barrel shifter should be set to $(B-A)$ mode $z$. When the DOP is increased to two, the barrel shifter should not only align $\alpha^{A}$ and $\alpha^{B}$, but also align $\alpha^{C}, \alpha^{D}$ and $\alpha^{A}$ with the shifting parameters of $(C-A)$ mode $z$ and $(D-A)$ mode $z$ in once iteration. The traditional design faces challenges to the implementation of high-DOP architectures.

Fig. 2 shows the behavior of check-node-unit processing (CNU). The traditional code and the corresponding architecture with only six CNU blocks are not suitable to simultaneously update two columns.

\subsection{Construction of parallel dispersed array QC-LDPC}

We adopt the Latin square algorithm to construct a base matrix $W^{a}$ of size $z \times z$, where $z$ is $2^{q}=64$. The entries $A_{i, j}$ of $W^{a}$ are the elements of $G F\left(2^{q}\right)$. For $0 \leq$ $i \leq 2^{q}-1$ and $0 \leq j \leq 2^{q}-1, A_{i, j}$ represents a submatrix that is either an all-zero matrix or a cyclic-shift of an identity matrix of size $2^{q}-1$. We can illustrate the construction of the code as follows.

- Step 1: Select the upper-left corner of matrix $W^{a}$ to construct a base matrix $H^{b}$ as in Eq. (4), of size $r \times s$, where $r \leq 2^{q}, s \leq 2^{q}$, and set $r=6$ and $s=60$.

$$
H^{b}=\left[\begin{array}{cccc}
A_{0,0} & A_{0,1} & \cdots & A_{0, s-1} \\
A_{1,0} & A_{1,1} & \cdots & A_{1, s-1} \\
\vdots & \vdots & \ddots & \vdots \\
A_{r-1,0} & A_{r-1,1} & \cdots & A_{r-1, s-1}
\end{array}\right]
$$




$H_{i}^{b}$
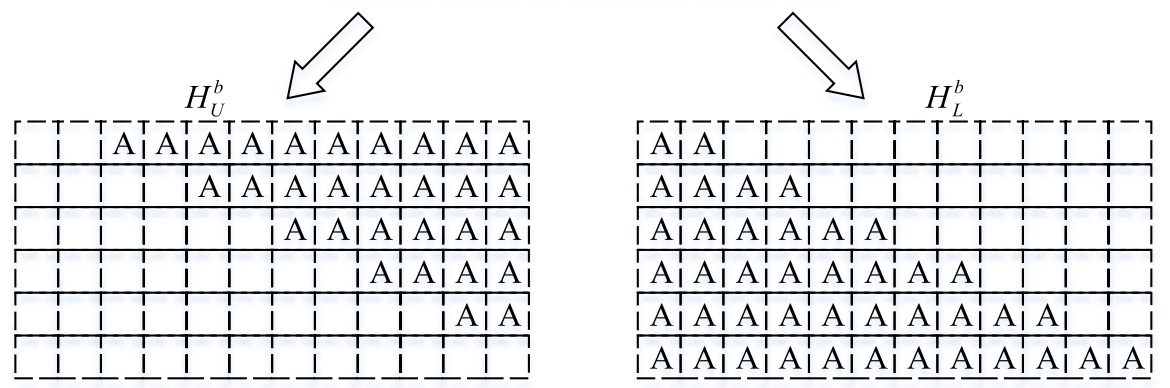

Fig. 3. Separation of matrix $H_{i}^{b}$

- Step 2: Divide the matrix into five submatrices by column, $H^{b}=\left[H_{0}^{b}, \ldots, H_{4}^{b}\right]$. Each submatrix as in Eq. (5) is a matrix of size $(r, p)$, and $p=2 r$.

$$
H_{i}^{b}=\left[\begin{array}{cccc}
A_{0,0} & A_{0,1} & \cdots & A_{0, p-1} \\
A_{1,0} & A_{1,1} & \cdots & A_{1, p-1} \\
\vdots & \vdots & \ddots & \vdots \\
A_{r-1,0} & A_{r-1,1} & \cdots & A_{r-1, p-1}
\end{array}\right]
$$

- Step 3: Instead of dividing $H_{i}^{b}$ diagonally [6], we separate the lower left corner and upper right corner of each $H_{i}^{b}$ by two-column-aligned strategy as Fig. 3 to generate the $H_{U}^{b}$ and $H_{L}^{b}$ with the same dimension of $H_{i}^{b}$. Except the element from the $H_{i}^{b}$, other elements of $H_{U}^{b}$ and $H_{L}^{b}$ are filled with zeros. The zeros represent a $\left(2^{q}-1,2^{q}-1\right)$ all-zero submatrix.

- Step 4: Apply array dispersion to expand $H_{U}^{b}$ and $H_{L}^{b}$ to a large matrix. Repeat and combine $H_{U}^{b}$ and $H_{L}^{b} t$ times to generate the diagonal-like structure matrix $H_{i}^{t}$ of size $(t \times r, t \times p)$ as in Eq. (6), where $t=5$. As in Step3, the extra positions are filled with zeros, and each represents an all-zero matrix of the same size as $H_{U}^{b}$ and $H_{L}^{b}$.

$$
H_{i}^{t}=\left[\begin{array}{ccccc}
H_{L}^{b} & 0 & 0 & 0 & H_{U}^{b} \\
H_{U}^{b} & H_{L}^{b} & 0 & 0 & 0 \\
0 & H_{U}^{b} & H_{L}^{b} & 0 & 0 \\
0 & 0 & H_{U}^{b} & H_{L}^{b} & 0 \\
0 & 0 & 0 & H_{U}^{b} & H_{L}^{b}
\end{array}\right]
$$

- Step 5: Connect the five diagonal-like matrices and obtain the final parity check matrix $H$ of size $(18900,1890)$ as in Fig. 4.

The Latin matrix $W^{a}$ satisfies row-column-constraint that no two rows and columns have 1 at the same place [11]. $H_{U}^{b}$ and $H_{L}^{b}$ decomposed from $H^{b}$ as in Fig. 3 do not violate the constraint. Similar to [6], there is only one $H_{U}^{b}$ and $H_{L}^{b}$ 


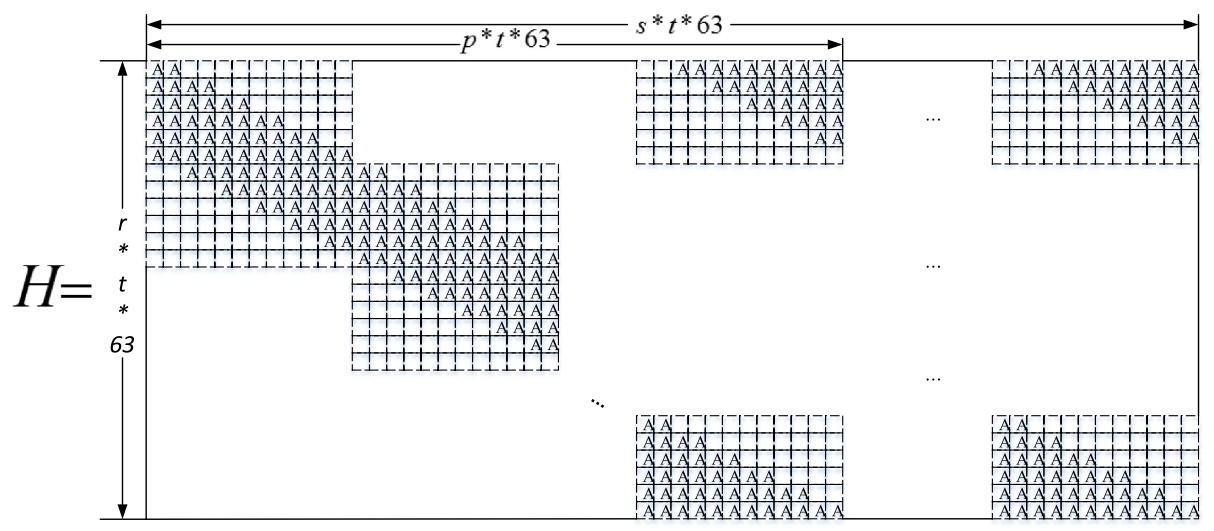

Fig. 4. $H$

in each column or row in expanded matrix. Therefore, matrix $H$ also contains no 4-cycle after array dispersion.

\section{Proposed architecture and the results of emulation}

\subsection{Overall parallel architecture}

Fig. 5 shows the architecture of the multi-column parallel decoder, and Fig. 6 shows a CNU and a data selector unit (DSU) in detail. There are only two VNU blocks, six CNU blocks, and 10 DSU blocks in total. Each CNU block contains 63 sorters units, 63 sign update units, and four barrel shifters for message and sign bits. A DSU contains a barrel shifter and 63 minimum selectors. Data flow starts from

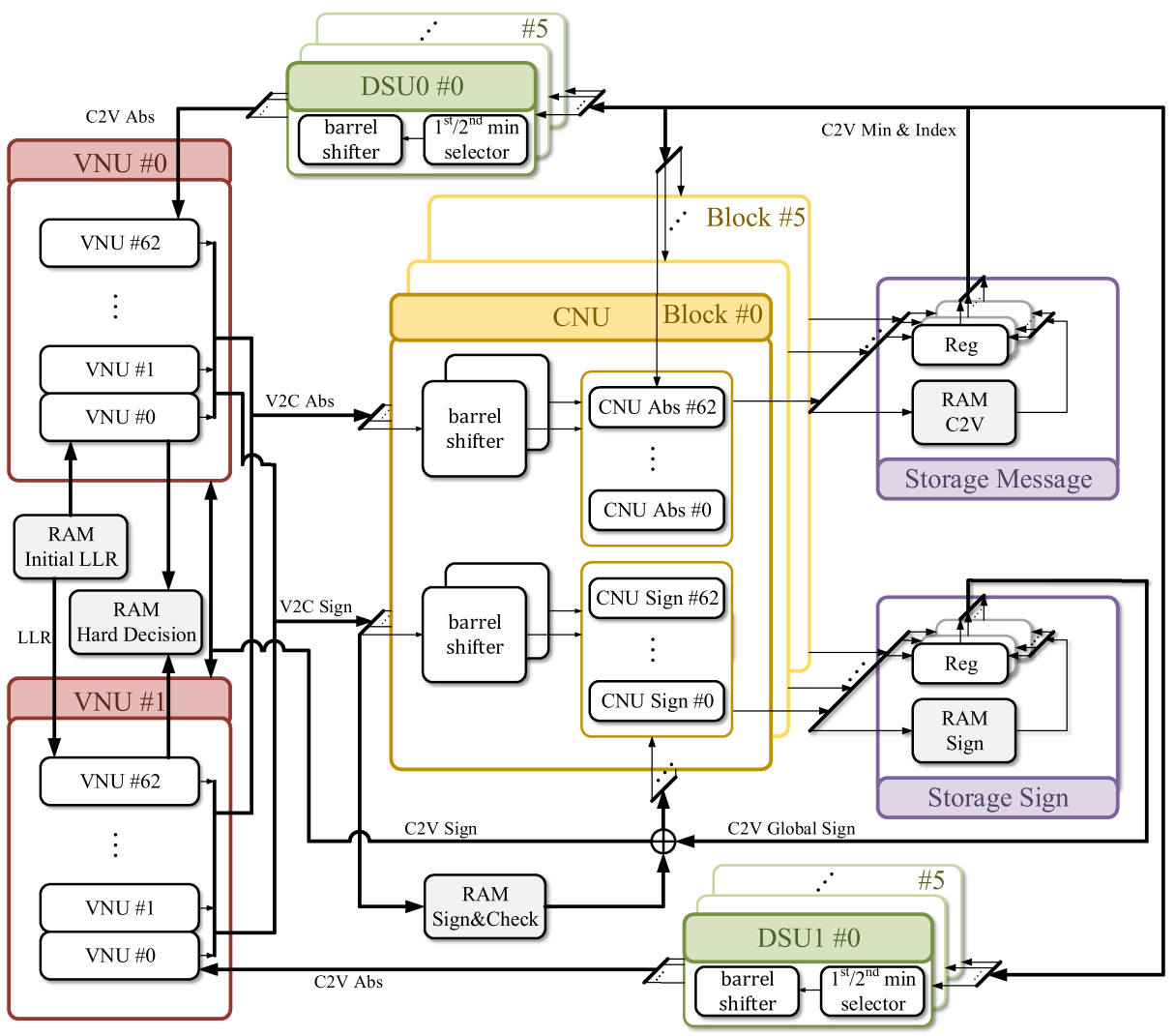

Fig. 5. Parallel decoder architecture for QC-LDPC 

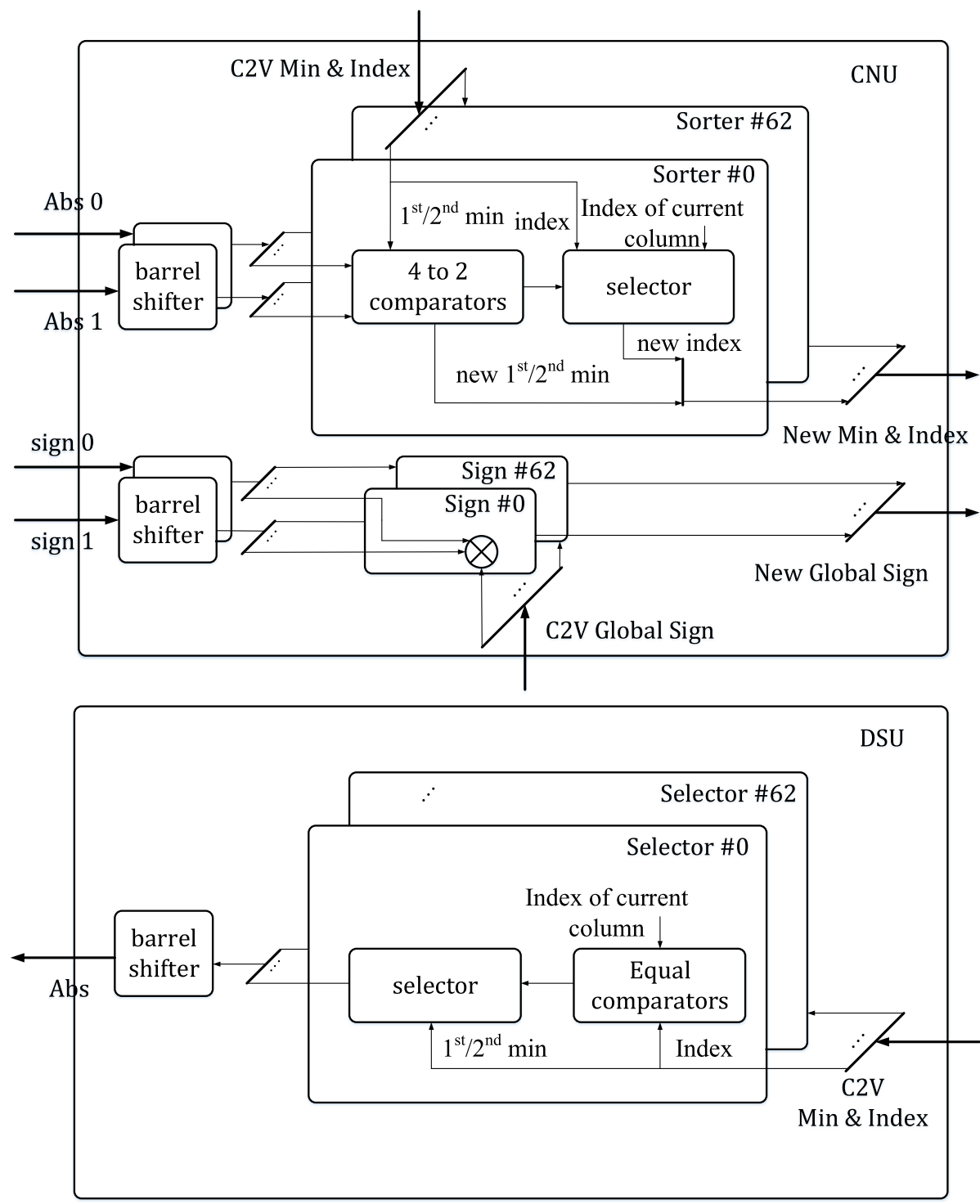

Fig. 6. Architecture of a CNU and a DSU block in detail

the storage component and passes through the DSU block, the VNU block, the CNU block, and is finally written back to memory.

As is shown in Fig. 6, the DSU updates the minimum value of the submatrix according to the entered $\mathrm{C} 2 \mathrm{~V}$ message and the index of the given column. Prior to being transferred to the $\mathrm{VNU}$, the selected minimum values are aligned in the barrel shifter the shifting value of which is equal to $A_{i, j}$. The $\mathrm{CNU}$ updates the $\mathrm{C} 2 \mathrm{~V}$ data according to the $\mathrm{V} 2 \mathrm{C}$ messages from the VNUs and the prior $\mathrm{C} 2 \mathrm{~V}$ messages. The shifting value of each barrel shifter in the $\mathrm{CNU}$ is $63-A_{i, j}$ to align the different V2C messages. Each CNU sorter contains one four-to-two comparator to generate the first and second minimum values. Each sign update unit gets a new global sign. Compared with [6], the proposed architecture costs twice selector resources and VNUs.

\subsection{Barrel shifters and storage memory}

In traditional architectures [6], barrel shifters are present only in CNUs, and need to process 19 bits, consisting of two C2V messages (three bits per value), two address 
Table I. Comparison of barrel shifters

\begin{tabular}{c|c|c|c}
\hline \multirow{4}{*}{ CNU } & & This work & TVLSI'16 [6] \\
\cline { 2 - 4 } & width & 4 & 19 \\
\cline { 2 - 4 } & number & $12 \times 63$ & $6 \times 63$ \\
\cline { 2 - 4 } & total & 3024 & 7182 \\
\hline \multirow{4}{*}{ DSU } & width & 3 & $\mathrm{~N} / \mathrm{A}$ \\
\cline { 2 - 4 } & number & $10 \times 63$ & $\mathrm{~N} / \mathrm{A}$ \\
\cline { 2 - 4 } & total & 1890 & $\mathrm{~N} / \mathrm{A}$ \\
\hline \multirow{2}{*}{ total } & & 4914 & 7182 \\
\hline
\end{tabular}

messages (six bits per value), and a global sign bit. The parallel architecture aligns the selected minimum value in the DSU first, and the V2C messages and sign bits in the CNU next. The width of each barrel shifter in the DSU is three bits message, and each barrel shifter in CNU processes three bits message and a sign bit. Table I lists a comparison of barrel shifters between the proposed architecture and that in [6].

Table II shows storage usage in the decoding architecture. Before decoding begins, the initial LLR message and the parity-check matrix are stored into a singleport RAM and ROM, respectively. Compared with [6], a hard-decision RAM and a sign-and-check RAM are twice the width and half the depth in the proposed architecture. The hybrid storage architecture containing a two-port RAM and six registers blocks is also adopted.

Table II. Width and depth of ROMs and RAMs

\begin{tabular}{c|c|c|c}
\hline & Type & Width (bit) & Depth \\
\hline Matrix ROM & single-port ROM & 72 & 150 \\
\hline Initial LLR RAM & single-port RAM & 252 & 150 \\
\hline C2V RAM & dual-port RAM & 1260 & 24 \\
\hline Sign-\&-check RAM & dual-port RAM & 882 & 150 \\
\hline Hard-decision RAM & single-port RAM & 126 & 150 \\
\hline
\end{tabular}

\subsection{Results of emulation}

We implemented the proposed architecture on the Xilinx Virtex UltraScale XCVU440 FPGA emulation platform. The maximum number of iterations was set to 20 and early termination was adopted.

For comparison, we constructed an $(18900,17010)$ LDPC code as in [6] without masking. The results of simulation with different DOPs are shown in Fig. 7. The FER and BER of both hard-one-bit and soft-two-bit decisions of the initial LLR were considered. The results show that the parallel architecture did not sacrifice correction performance in terms of BER.

Table III lists comparisons with several related decoding architectures implemented in the TSMC $90 \mathrm{~nm}$ library. The results show that the proposed architecture 


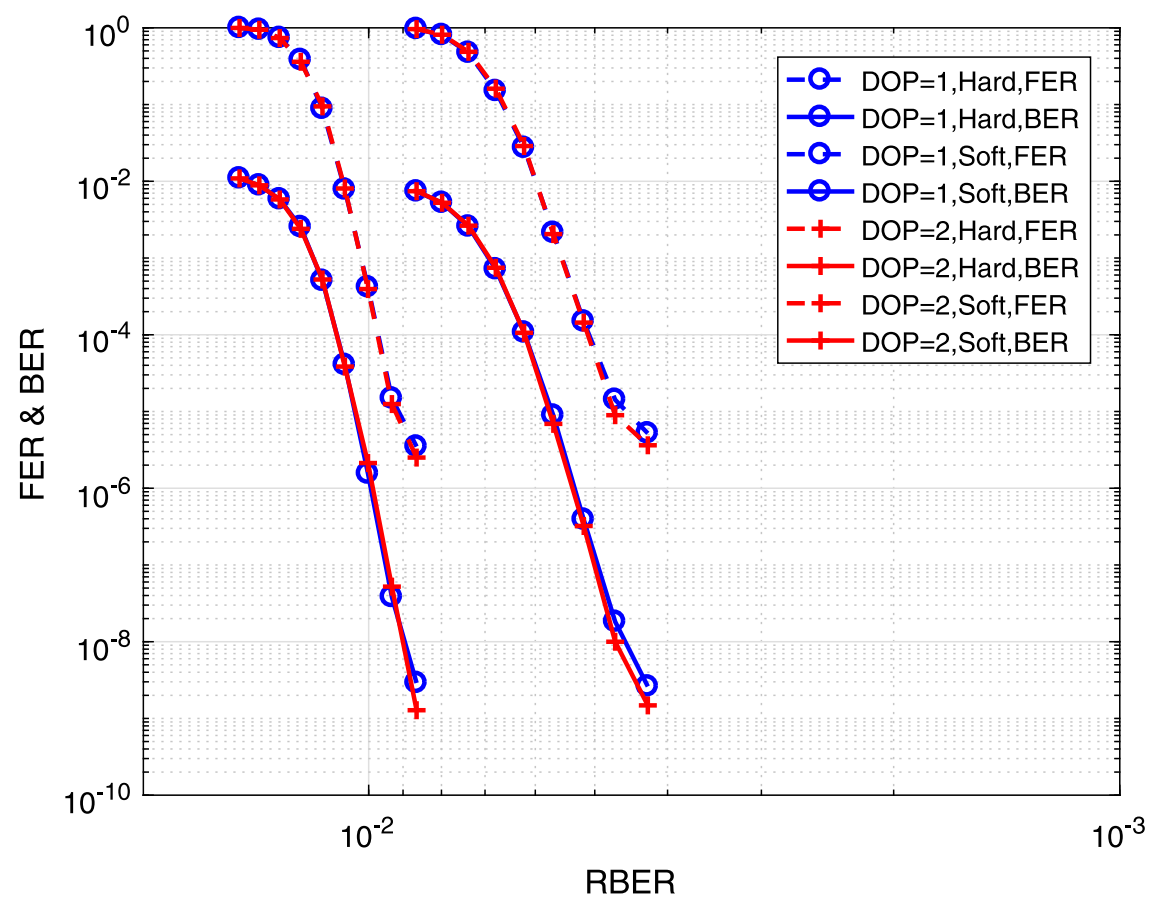

Fig. 7. Performance comparison in terms of FER and BER between $\mathrm{DOP}=1$ and DOP $=2$ of the proposed $(18900,17010)$ QCLDPC code over the AWGN channel. The number of quantization bits of the intermediate message was 4 and the scaling factor was 0.75

Table III. Comparision results

\begin{tabular}{c|c|c|c|c}
\hline & $\begin{array}{c}\text { This work } \\
\text { [Proposed] }\end{array}$ & $\begin{array}{c}\text { [6] K. Ho } \\
\text { TVLSI'16 }\end{array}$ & $\begin{array}{c}\text { [11] W. Shao } \\
\text { TCAS-II'17 }\end{array}$ & $\begin{array}{c}\text { [7] H. Lee } \\
\text { TCAS-I'17 }\end{array}$ \\
\hline Impl. & post-layout & post-layout & post-layout & post-layout \\
\hline Schedule & Shuffled & Shuffled & Shuffled & Layered \\
\hline Iterations & 6 & 6 & 6 & 8 \\
\hline Quant. & 4 & 4 & 4 & 5 \\
\hline Technology & $90 \mathrm{~nm}$ & $90 \mathrm{~nm}$ & $90 \mathrm{~nm}$ & $90 \mathrm{~nm}$ \\
\hline Code length & 18900 & 18900 & 18300 & 18396 \\
\hline Code rate & 0.9 & 0.9 & 0.897 & 0.905 \\
\hline Freq. (MHz) & 166 & 166 & 200 & 166 \\
\hline Gate count & $732 \mathrm{k}$ & $520 \mathrm{k}$ & $410 \mathrm{k}$ & $926 \mathrm{k}$ \\
\hline Area (mm $\left.{ }^{2}\right)$ & 2.93 & 2.56 & 1.44 & 4.19 \\
\hline $\begin{array}{c}\text { Throughput } \\
(\text { Gbps) }\end{array}$ & 3.49 & 1.58 & 1.83 & 4.25 \\
\hline$*$ TAR & 1.19 & 0.62 & 1.27 & 1.01 \\
\hline
\end{tabular}

*Throughput-to-area ratio, TAR $=($ Throughput $) /($ Area $)$

achieved a throughput of $3.49 \mathrm{~Gb} / \mathrm{s}$ under an average number of six iterations and a clock frequency of $166 \mathrm{MHz}$. For fair comparison, we considered normalized throughput by computing the throughput-to-area (TAR). Compared with related work, the TAR of the proposed architecture was improved from 0.62 to 1.19. 


\section{Conclusion}

In this paper, a $2 \mathrm{~KB}$-long QC-LDPC was proposed based on the Latin square and a new array dispersion method. With its diagonal-like structure, the parallel architecture reduces the number of barrel shifters compared with the traditional design. The results of simulations show that the TAR of the proposed codes is significantly improved.

\section{Acknowledgments}

This work was supported by the National Key $R \& D$ Program of China (No. 2016YFA0202102), the National Natural Science Foundation of China under Grant No. 61370040, the project on the Industry Key Technologies of Jiangsu Province, BE2017153, Shenzhen science and technology project JCYJ20170818110714340 and a Project Funded by the Priority Academic Program Development of Jiangsu Higher Education Institutions (PAPD). 